\title{
Advances in Study of Mechanical Properties of Gas Hydrate-Bearing Sediments
}

\author{
Lu X.B*, Zhang X.H and Wang S.Y
}

Institute of Mechanics, Chinese Academy of Sciences, Beijing 100190, China

\begin{abstract}
Gas hydrate (GH) is defined as the crystalline solid, or clathrate hydrate, which are formed by some kinds of low mass molecular gases, such as methane, carbon dioxide, and hydronitrogen, with water at relatively high pressure and low temperature conditions. Gas hydrate-bearing sediments (HBS) are some sand, clay and mixed sediment containing gas hydrates. Advances in the study on the mechanical properties of HBS are summarized mainly in aspects of the laboratory test, in-situ investigation, and theoretical model. Firstly, the main factors are discussed including the structure of GH, formation method and matrix characteristics of HBS; Secondly, progress on the laboratory tests and results are discussed, which mainly includes the tri-axial tests with the natural and synthesized HBS samples, the acoustic tests for measuring the elastic coefficients, the tests for investigating the effects of main factors such as the gas and water contents and soil types on the strength of HBS. Thirdly, for in-situ investigations, including the geophysical surveying, in-situ tests (such as downhole tests) and results are summarized; Fourthly, several theoretical models for estimating the mechanical properties of HBS are introduced; At last, the emphases and the tendency in the future study on the mechanical properties of HBS are discussed.
\end{abstract}

Keywords: Gas hydrate sediments, In-situ test, Laboratory test, Theoretical model.

\section{INTRODUCTION}

Gas hydrates $(\mathrm{GH})$ are defined as the crystalline solid, or clathrate hydrate, which are formed by some kinds of low mass molecular gases, such as methane, carbon dioxide, and hydronitrogen, with water at relatively high pressure and low temperature conditions (Fig. 1). HBS is sediments of soil or rock mixed with $\mathrm{GH}$, which usually exists in deep water or permafrost.

Humphrey Davy is the first man to discover GH in 1810. The investigation of GH was started until 1934 when the American scientist E. G. Hammershmer found that the blockage of the oil/gas pipelines was mainly due to the formation of GH. In 1960's, the former Soviet Union exploited $\mathrm{GH}$ in the permafrost area--the Siberia Misoyaha [1]. Since 1970's, ODP (Ocean Drilling Program) [2] was processed supported by several countries.

Currently, scientists from more than 30 countries are studying HBS. HBS has been found in more than 100 places in more than 70 countries and, more than 80 places locate in the ocean [3] $164 \mathrm{~m}^{3}$ methane and $0.8 \mathrm{~m}^{3}$ water can be produced from $1 \mathrm{~m}^{3} \mathrm{GH}$ after dissociation. Thus it is a kind of important potential resource [4].

However, HBS is easy to dissociate once the temperature or pressure changes. The excess pore pressure induced by the dissociation of GH not only destroys the connection among the grains of HBS, but also leads to bubbles due to the super-

*Address correspondence to this author at the Institute of Mechanics, Chinese Academy of Sciences, Beijing 100190, China; Tel: 8610-82544190; Fax: 861062561284; E-mail: xblu@imech.ac.cn saturation of the natural gas in the solution which can cause the expansion of pores and the decrease of the effective stress and the strength. So, the sediments tend to be of lowstrength and stiffness and unsaturated and underconsolidated, which is a large threat to the stability of ocean beds.

At present, the lack of resources has become a global problem, so the exploitation of $\mathrm{GH}$ attracts many countries. The USA and Japan planned to start the business exploitation of GH in 2015 and 2016, respectively [5].

There are four media coexist in HBS: gas, soil or rock skeleton, water and solid gas hydrate. The saturation degrees of water and gas change with the dissociation of GH. The mechanical properties of HBS such as the strength and modulus change accordingly.

It is very important to clarify the mechanical parameters of HBS since they are the basis for all of the studies and practices of HBS meanwhile they are very lack.

The methods for obtaining the mechanical properties of HBS are mainly the field survey, laboratory test, theoretical analysis. The measurement methods and techniques in field survey include seismic geophysical method, drilling core method, sonic wave method, well-logging method etc. The laboratory test is mainly to measure the stress-strain curves, strength, decomposition characteristics and thermal conduction properties of HBS. The theoretical analysis is to present models for describing the elastic constants and the contents of HBS based on different hypotheses.

In fact, the dissociation of HBS contains four kinds of physical processes: stress transformation, seepage, phase translation and thermal conduction. The characteristic times 


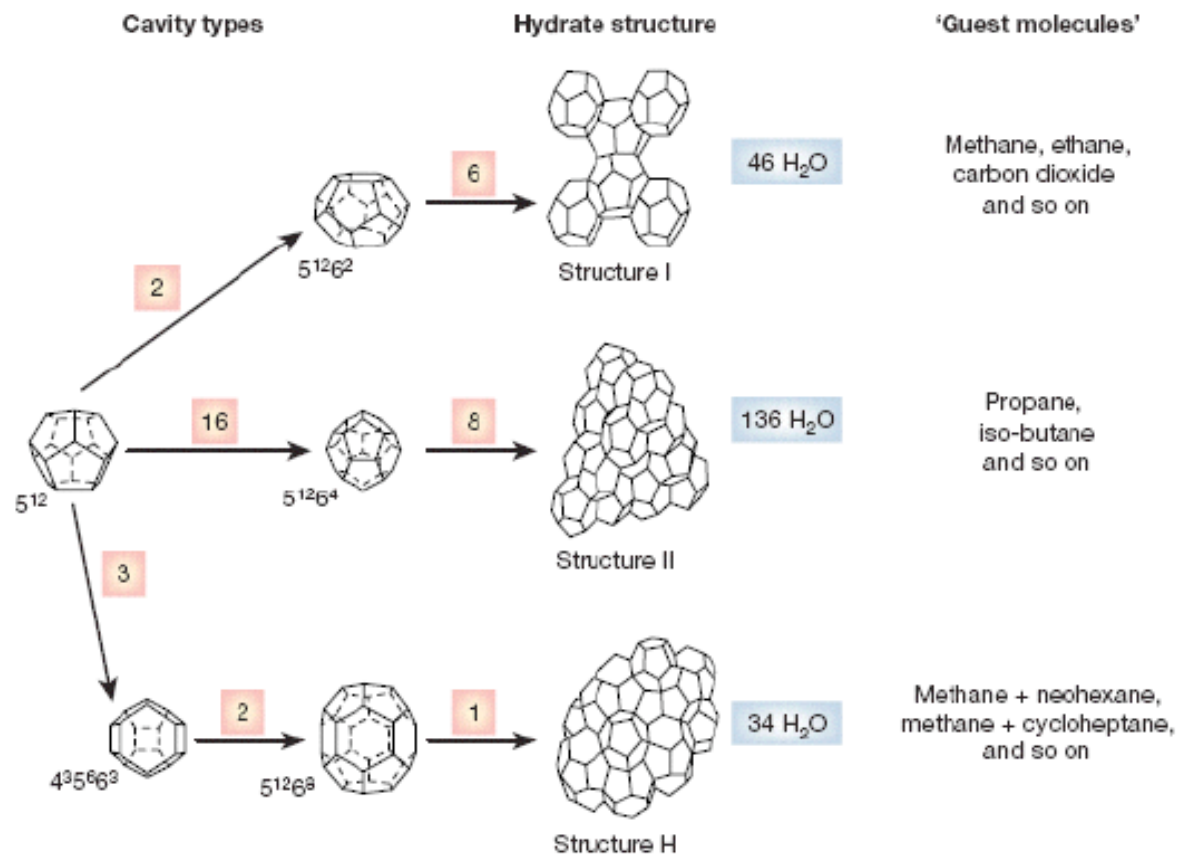

Fig. (1). Gas hydrate crystal structure.

in the four processes are as follows: characteristic time of elastic wave propagation $t_{\mathrm{e}}=1 /(\mathrm{E} / \rho)^{1 / 2}$, seepage characteristic time $t_{p}=\mu_{g} l^{2} / k_{g} p_{e}$, characteristic time of phase translation $\rho_{h} /\left[k_{d}\left(p_{e}-p_{g}\right) A_{s}\right]$, heat conduction characteristic time $\mathrm{t}_{\mathrm{c}}=\rho \mathrm{Cl}^{2} / \lambda$, and here, E, $\rho, \mathrm{C}, 1, \lambda, \mu_{\mathrm{g}}, \mathrm{k}_{\mathrm{g}}, \mathrm{p}_{\mathrm{e}}, \rho_{\mathrm{h}}, \mathrm{k}_{\mathrm{d}}, \mathrm{p}_{\mathrm{g}}, \mathrm{A}_{\mathrm{s}}$ are elastic modulus, density of matrix, specific heat, characteristic length, thermal conductivity, methane gas viscosity, gas permeability, pressure at phase equilibrium, density of hydrate, coefficient of hydrate dissociation and dissociation area respectively. The ratios of these four characteristic parameters are about $10^{9}: 10^{6}: 10^{6}: 1$. That means, the problem can be regarded as four independent processes [6].

The main factors are the density, permeability, heat conduction and latent heat [7]. These factors are related with the type and saturation degree of $\mathrm{GH}$, sedimentation, the proportion of microbe, the distribution and cementation, the proportion of microbe and bacilli and microfossil. Generally, the larger the saturation degree of GH and the higher the cementation are, the higher the strength of HBS is. The sedimentation characteristics of the skeleton including the lithology, sedimentary structure affect much on the mechanical properties of HBS.

In this paper, the effects of $\mathrm{GH}$ formation and the pore scale and the distribution of GH in sediment on the mechanical properties of HBS are discussed first. Then the advances in the mechanical properties of HBS determined in laboratory and in-situ investigation are reviewed. At last, the application and prospect are discussed.

\section{MAIN FACTORS RELATIVE TO MECHANICAL PROPERTIES}

\subsection{Structure of Gas Hydrate}

HBS can be divided into three types by the structures: Type-I、 Type-II、 Type-H (Fig. 1). HBS of Type-I is cubic crystal, Type-II prismatic cubic crystal, Type-H hexagonal.
The theoretical structures of Type-I and Type-II HBS are $\mathrm{S}_{2} \mathrm{~L}_{6} \cdot 46 \mathrm{H}_{2} \mathrm{O}$ and $\mathrm{S}_{16} \mathrm{~L}_{8}^{\prime} \cdot 136 \mathrm{H}_{2} \mathrm{O}$ respectively, in which $S$ indicates one multiple crystal cage with 20 nodes (20cage), $L$ indicates one multiple crystal cage with 24 nodes (24-cage), $L^{\prime}$ one multiple crystal cage with 28 nodes (28cage). The cubic ratio is 20 -cage: 24 -cage: 28 -cage $=1.91$ : 1.43: 1 and the equivalent sphere diameters are 4.18, 3.79, $3.37 \dot{A}$ respectively. At $100 \mathrm{~K}$, the average crystals' volumes are $158.8 \dot{A}^{3}, 227.8 \dot{A}^{3}, 303.8 \dot{A}^{3}$, respectively. The diameter of $\mathrm{CH}_{4}$ is $0.4-0.5 \mathrm{~nm}$ [1].

\subsection{Formation of HBS}

The formation of $\mathrm{GH}$ in sediment is affected by the pore scale, the physical and chemical characteristics of the grain surface, grain series, grain shape, permeability etc., which is obviously different with that of cubic GH.

Methane hydrate is stable only at cold temperature and high gas pressure conditions. Thus pristine samples of natural HBS are difficult to retrieve and make measurements on. Pressure coring has been performed successfully at a few locations, retrieving the most representative samples so far; however, these samples are not readily available for all studies or, at some cases, are not amenable to the needed study. Because of this difficulty, Formation technique for HBS is required for laboratory study [8].

Even for water in contact with hydrate at $4^{\circ} \mathrm{C}$, there are 750 water molecules per methane molecule, as compared to 6 water molecules per methane molecule required in the methane hydrate structure. So it is very difficult to synthesize methane hydrate. However, several kinds of methods have been discovered.

Different laboratory methodologies for synthesizing methane hydrate can result in different hydrate habits and hence different physical properties for identical sediments 


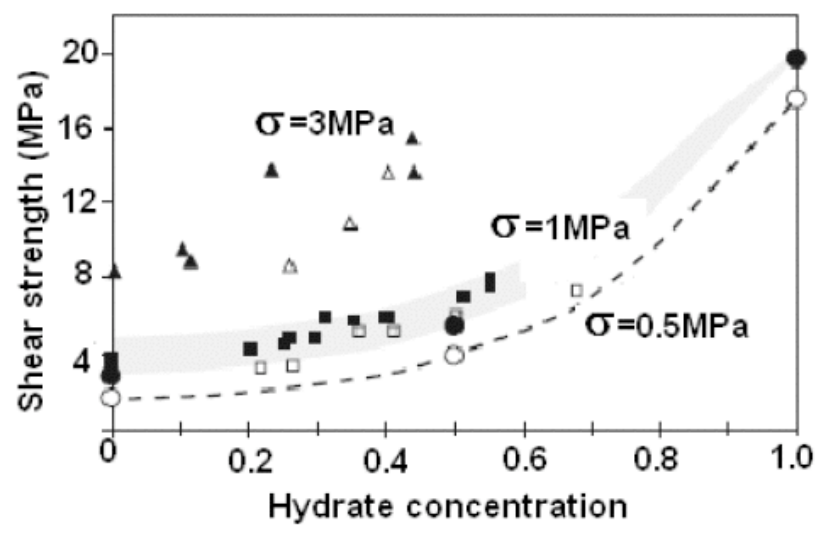

Fig. (2). Relation between the hydrate saturation and the shear strength of HBS (From Literature [15]).

with equal hydrate saturations. These laboratory methods produce different pore-scale growth habits $[9,10]$. The often used methods are the following four. Dissolved Gas Method: water saturated with a hydrate-forming gas is circulated through sediment that is held within the hydrate pressure and temperature stability field. The hydrate growth rate is limited by the concentration of hydrate former in the water. The dissolved gas method is limited to forming hydrate saturations Sh below $60 \%-70 \%$ for which water remains a percolating phase and can continue to circulate. Partial Water Saturation Method: Soil grains are mixed with a limited amount of water (or by introducing the methane as bubbles into the fully water saturated soils) and packed to form a partially water-saturated sediment. The system is pressurized with methane gas and cooled into the stability field to promote hydrate formation. Depending on the initial water saturation, this method can take just a few days to form hydrate-bearing sediments [8,11]. This method leads to preferential hydrate formation at contacts and stiffening of the sediment framework [12]. Ice-Seeding Method: cooled soil grains are mixed with small ice grains. The mixture is pressurized into the hydrate stability field with methane. The temperature is slowly increased. Hydrate nucleation is facilitated by the existing ice lattice, and hydrate can grow rapidly from the water liberated as the ice melts $[13,14]$. Depending on the gas pressure, this method can be run to completion within a few days. Hydrate Premixing Method: Granular methane hydrate is prepared first and then the methane hydrate granules are mixed with sediment at very low temperature and consolidated at the target effective stress for a few hours.

By using dissolved gas method, heterogeneous nucleation occurs anywhere on the particle surface, with subsequent growth into the pore space (Pore-filling type). The most pronounced increase in strength is expected when the hydrate saturation exceeds $40 \%$ (Fig. 2)[15]. If the hydrate saturation exceeds $25-40 \%$, pore-filling type HBS naturally turns into another type: load-bearing-type. By using the partial water saturation method, methane hydrate preferential forms at contacts of particles and stiffens the sediment framework (cementation-type). By using ice-seeding methods, the relative size and volume fraction of particles and methane hydrate determine their relative load-bearing contribution to the sediment framework. By using the hydrate premixing method, the relative load-bearing contribution to the sedi- ment framework depends on their relative size of particles and methane hydrate. However, the formation patterns of naturally occurring hydrate are varied, with an observable distinction between disseminated, pore filling hydrate in coarse sands compared to veined or nodule-type occurrences in fine-grained sediments. Therefore, suitable synthesis method must be chosen if one want to investigate the properties of in-situ HBS in laboratory [16].

\subsection{Characteristics of Matrix and Formation Methods on Mechanical Properties}

The pore-scale habits of hydrates, hydrate formation techniques of $\mathrm{GH}$ exerts a strong control on the macroscale physical properties of HBS. The three most commonly discussed hydrate habits are shown in the section 2.1: The first habit is pore-filling. Hydrates nucleate on sediment grain boundaries and grow freely into pore spaces without bridging two or more particles together. In this case, hydrate primarily affects the pore fluid bulk stiffness and fluid conduction properties [17]. The second habit is load-bearing. Hydrate bridges neighboring grains and contributes mechanical stability to the granular skeleton by becoming part of the load-bearing framework. The third habit is cementation. Hydrate cements intergranular contacts. Even a small amount of hydrate can dramatically increase the sediment shear and bulk stiffness by bonding adjacent grains together [18].

The mechanical properties of hydrate-bearing sediments at low hydrate concentration (probably $<40 \%$ of pore space) appear to be determined by stress-dependent soil stiffness and strength. At high hydrate concentrations $(>50 \%$ of pore space), the behavior becomes more independent of stress because the hydrates control both stiffness and strength and possibly the dilative tendency of sediments by effectively increasing interparticle coordination, cementing particles together, and filling the pore space. The cementation contribution to the shear strength of hydrate-bearing sediments decreases with increasing specific surface of soil minerals. The lower the effective confining pressure is, the greater the impact of hydrate formation on normalized strength is. Increasing confining pressure leads to increases in interparticle coordination, contact area and frictional resistance, and consequent increases in sediment framework stiffness. However, hydrate controls the bulk stiffness of HBS in the presence of high concentrations of hydrate, and confining pressure has a negligible impact in these cases. In the absence of hydrates, the shear strength is of frictional nature and depends on confining pressure.

\section{MECHANICAL PROPERTIES}

\subsection{Stress-Strain Relation and Strength Obtained from Laboratory}

Sediment strength is a combination of the cohesive resistance, c, and effective stress-dependent frictional resistance described by the friction angle, $\varphi$, which includes resistance to sliding between particles, particle rearrangement, and particle crushing. The two contributions to shear strength are captured in the Coulomb failure criterion which relates the shear stress at failure, $\tau_{f}$, to the normal effective stress, $\sigma_{e}$, acting on the failure plane. 
Table 1. Comparison of Thermal Parameters of THF Hydrate and Methane GH

\begin{tabular}{|c|c|c|}
\hline & THF Hydrate & Methane GH \\
\hline \hline Thermal conductivity $/ \mathrm{m} / \mathrm{k}$ & $0.45 \sim 0.54$ & $0.4 \sim 0.6$ \\
\hline Specific heat $/ \mathrm{kJ} / \mathrm{kg} / \mathrm{K}$ & 2.123 & $1.6 \sim 2.7$ \\
\hline Density $/ \mathrm{kg} / \mathrm{m}^{3}$ & 997 & 913 \\
\hline Latent heat $/ \mathrm{kJ} / \mathrm{kg}$ & 270 & $-1050^{*} \mathrm{~T}+3527000$ \\
\hline Thermal diffusivity $/ \mathrm{m}^{2} / \mathrm{s}$ & $2 \times 10^{-8} \sim 4 \times 10^{-8}$ & \\
\hline
\end{tabular}

Note: (T-temperature)

Damping of hydrate-cemented sand was found to be much higher than that of sand specimens with the same equivalent water content. A peak damping ratio was found at about $3-5 \%$ hydrate content, both in torsion and in flexure[16,19]. When HBS is deformed in compressional creep tests, at steady state, typically reached in a few percent strain, methane hydrate exhibited high strength. In terms of the standard high-temperature creep law, $\dot{\varepsilon}=A \sigma^{n} e^{-\left(E^{*}+P V^{*}\right) R T}$, the rheology is described by the constants $A=10^{8.55} \mathrm{MPa}^{-n} \mathrm{~S}^{-1} \mathrm{~A}=108.55, \mathrm{n}=2.2, \mathrm{E}^{*}=90,000$ $\mathrm{J} \mathrm{mol}^{-1}$, and $\mathrm{V}^{*}=19 \mathrm{~cm}^{3} \mathrm{~mol}^{-1}$. At temperature just below the ice point, methane hydrate at a given strain rate is over 20 times stronger than ice, and the contrast increases at lower temperature. Inelastically deforming methane hydrate apparently causes it to dissociate, even at pressures and temperatures well within its phase stability field. There are two sets of observations that indirectly suggest that free water is generated during the ductile deformation of methane hydrate. The first is pronounced strain softening at high-strain rates, and the second is a greater swelling of sample diameter at the end away from the pore pressure line [20].

The study on the property of the sediments mainly composed of clay and silt particles showed that the clay minerals (predominantly illite) largely control sediment characteristics. The strength, stiffness, and porosity of the near-seafloor sediments are governed not by overburden vertical effective stress, but rather by interparticle forces arising from the interaction of the ionic pore fluid with the high specific surface of sediment grains. Pore fluid conductivity was in some cases twice the conductivity of sea water. The highly conductive pore fluids led to the dominance of interparticle electrical forces over effective stress in controlling the mechanical properties of near-seafloor sediments. The severe loss of sediment strength upon hydrate dissociation reflects (a) the increase in pore pressure associated with the transformation of load-bearing hydrate to water and dissolved gas and (b) the destruction of clay structure due to the increase in interparticle electrical repulsion as a result of pore water freshening associated with hydrate dissociation and due to gas expansion. High anomalous strength and shear stiffness were associated with the presence of carbonates [21].

To avoid the dissociation of GH during moving samples, someone uses tetrahydrofuran (THF) hydrates to study the mechanical properties of HBS because the physical and mechanical properties of THF hydrate-bearing sediment are similar to HBS [22](Table 1). Though the polar nature of the THF molecule compared to the nonpolar nature of the methane molecule have been raised. This polarity difference between THF and methane loses relevance in the context of hydration processes because the large size and structure of the THF molecule significantly weaken polarity-based ionic interactions between water and THF. The main advantage of THF relative to methane is its complete miscibility in water and the THF liquor can form hydrate at the conditions of 1 atm and temperature less or equal to $4^{\circ} \mathrm{C}$, which enables relatively rapid, homogeneous synthesis of THF hydrate and control of the hydrate volume fraction in sediments. However, THF hydrate does not dissociate to a gaseous phase, meaning many production-related processes are difficult to study with this analog. Within these limitations, THF hydrate is allowed the study of a wide range of material parameters that provide valuable insight to natural hydrate-bearing sediment $[19,23]$.

Winters et al. [24-26] studied the mechanical properties of HBS by using the following three kinds of samples: (1) samples coring from the Malik21-38 well, (2) the synthesized samples with Ottawa sand, (3) the samples containing water ice without GH. The resonance and triaxial experimental results showed that both the strength and the compressive wave speed of the HBS in-situ and the remoulded ones were larger than that of samples without GH. The increment depended on the saturation degree, the distribution of $\mathrm{GH}$ and the properties of sediments (Figs. 3, 4).

Hyodo's experiments showed that the routine of temperature and pressure during the formation of HBS had few effects on the strength of HBS with sand as the skeleton [23]. The mechanical properties of HBS depend on the temperature, back pressure, effective confining pressure and saturation degree of GH. Even when the shear stain arrived at $50 \%$ at which condition the cementation force was believed to lost, the residual strength is still higher than that of Toyoura sand. In other words, the increase of GH saturation degree leads to the increase of residual strength. During the dissociation of $\mathrm{GH}$, the volume strain is dilatant whether the effective confining pressure increases or decreases when no shear stress is applied on the sample. The changes of volume strain depended on whether or not the critical void ratio is achieved and on the saturation degree when the shear stress is applied on. The cementation of grains by $\mathrm{GH}$ has large effects on the shear modulus but few effects on the volume modulus. The ratio of the volume modulus to shear modulus changes from 15 30 (depended on the effective stress) to 2 


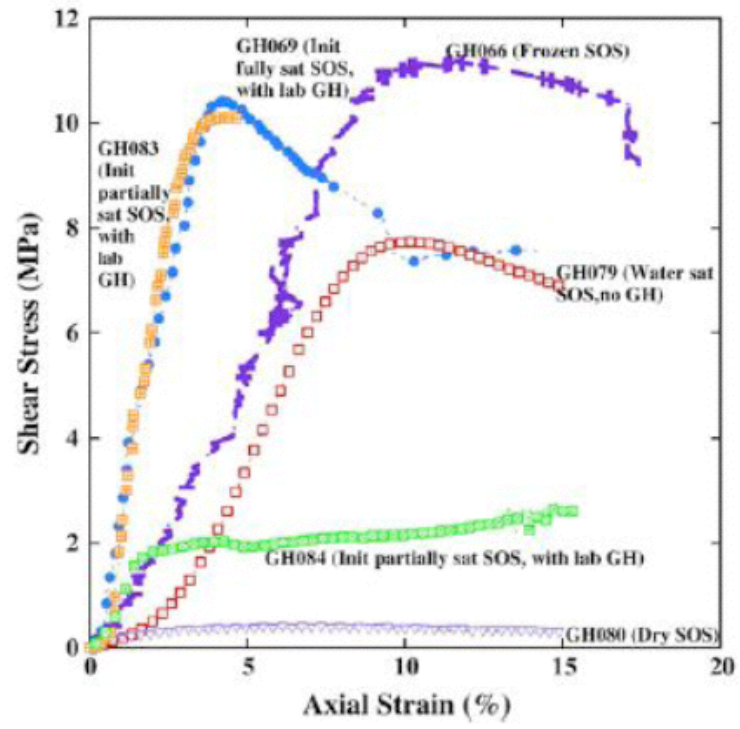

Fig. (3). Shear-stress versus axial strain plots for sieved Ottawa sand specimens containing various materials in pore space [25].

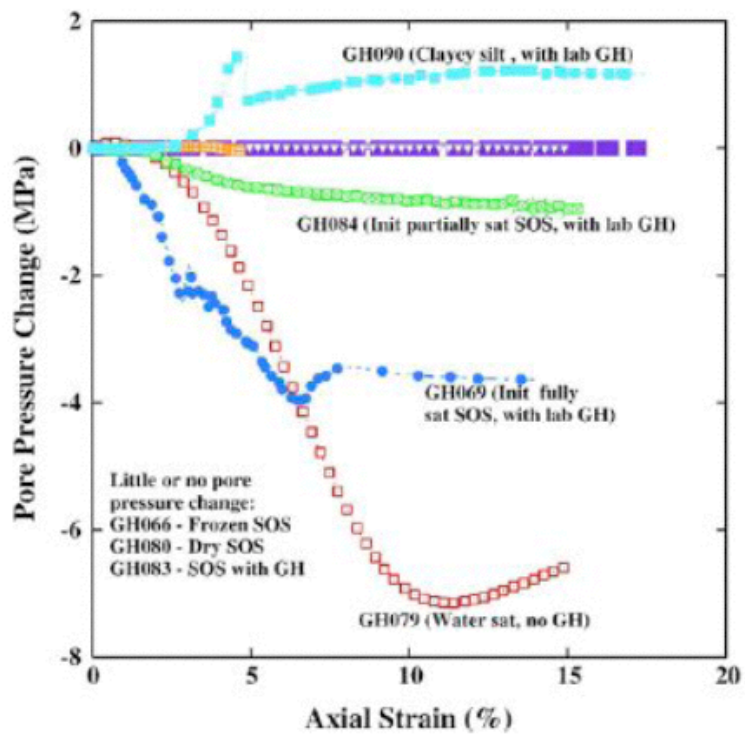

Fig. (4). Pore pressure changes versus strain results from Ottawa sand clayey silt [26].

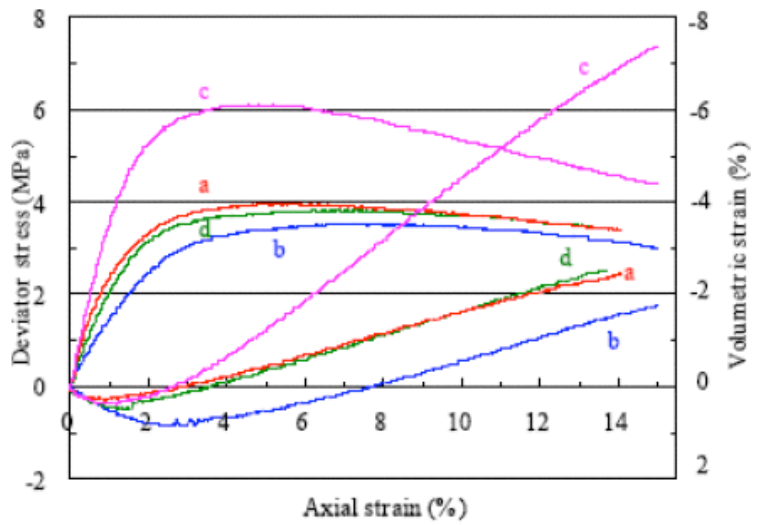

Fig. (5). Stress-strain relationships of natural gas hydrate core.

(when the saturation degree is 20\%). The damping ratio is higher when the grains are cemented by GH than that with-

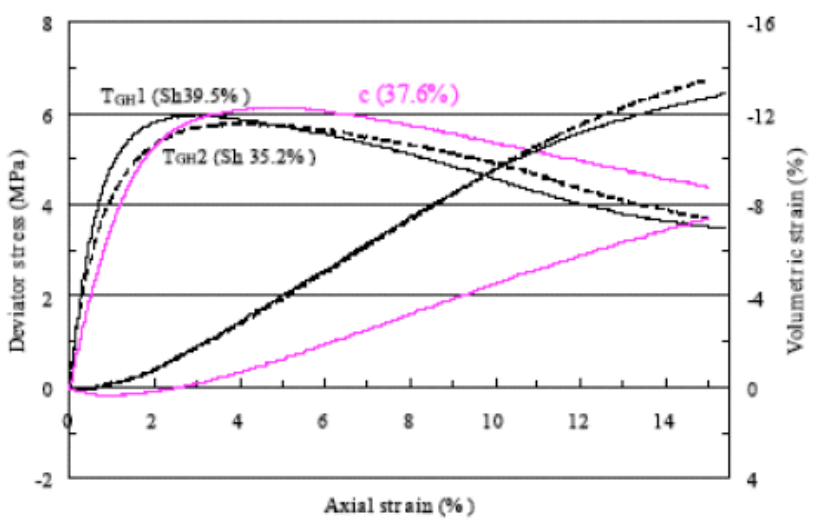

Fig. (6). Stress-strain relationships of natural gas hydrate core and synthetic GH sand.

out $\mathrm{GH}$ and, the maximum occurs when the saturation degree of $\mathrm{GH}$ is $3-5 \%$ [19].

The strength of in-situ samples is almost the same as that of the synthesized samples, but the relations of volume strain $\sim$ axial strain and stress $\sim$ strain are different mainly due to the differences of initial void ratio and grain series (Figs. 5, 6). The average tangential elastic modulus of the former is about $200 \mathrm{MPa}$ lower than that of the latter's. The Poisson's ratio of in-situ samples ranges from 0.1 to 0.2 , while it is $0.05 \sim 0.22$ for synthesized samples $[27,28]$. Thus it is practical to study the properties of HBS in laboratory. The strength increases with the rise of the strain rate, confining pressure, and with the decrease of the initial void ratio [29-31].

The stress-strain and shear strength characteristics of four kinds of deposits (ice, tetrahydrofuran (THF) hydrate, $\mathrm{CO}_{2}$ hydrate and methane hydrate $(\mathrm{MH})$-bearing sediments)and the effects of gas hydrate and ice on the strength of deposit show that these four deposits behaved as plastic failure. The deposits with the same saturation of gas hydrate have different strengths when the kinds of hydrate are different. After dissociation of hydrate, the strength of the sediment decreases greatly. For an example, if the hydrate sediment with fine silty sand as the skeleton and the hydrate saturation is $86 \%$, the strength after dissociation of hydrate is only $1 / 10$ of the initial value, while it is $1 / 2$ if the saturation is $30 \%$ [32, 33].

Though the cohesion of pure water ice is a little smaller than that of pure THF hydrate, while the internal friction angle is a little larger, the cohesion and internal friction angle of THF hydrate-bearing sediment are both larger than that of ice-bearing sediment when the saturations in these two kinds of sediments. With the increase of total saturation of ice and $\mathrm{MH}$, the internal friction angle of HBS decreases a little while the cohesion increases obviously. When the sum of methane hydrate and ice concentration in MH-bearing sediment is the same as that in ice sediment, the cohesion of $\mathrm{MH}$-bearing sediment is 2 times lower than that of icebearing sediment, while the internal friction angle is a little smaller (Fig. 7). The strength after dissociation of hydrate is only $1 / 2$ of the initial value when the hydrate saturation is $30 \%$ and it is only $1 / 10$ when the hydrate saturation is $86 \%$ (Fig. 8). The result is similar to that obtained by Winters et al. [25]. Under dynamic loads, longer duration is required for the sediment after dissociation of hydrate to liquefy relative 


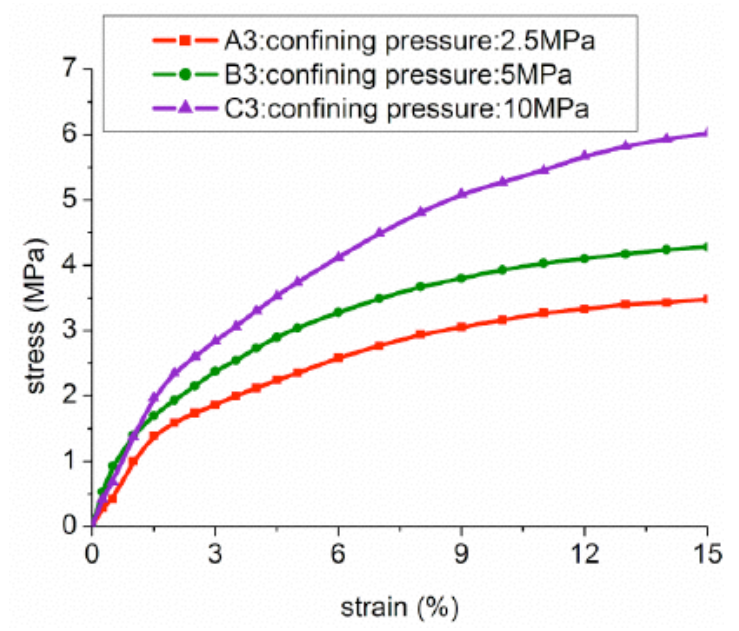

(a) stress-strain curve

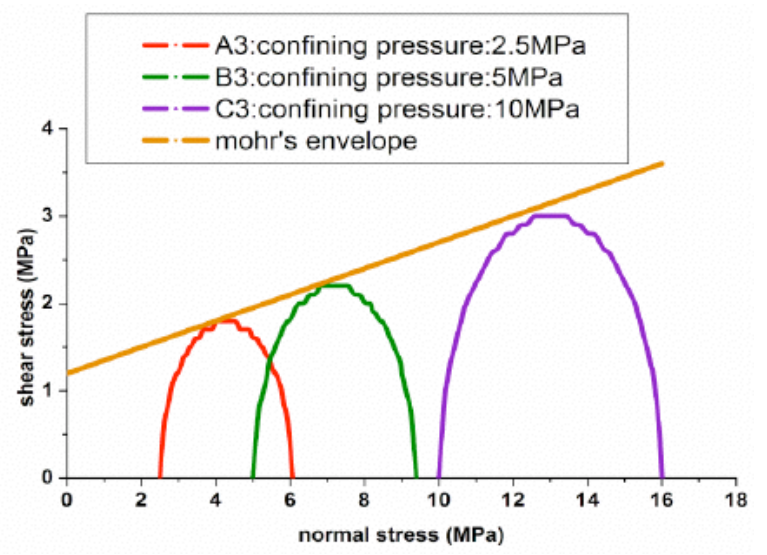

(b) Mohr circle

Fig. (7). Stress-strain curves and Mohr circles for MHS.

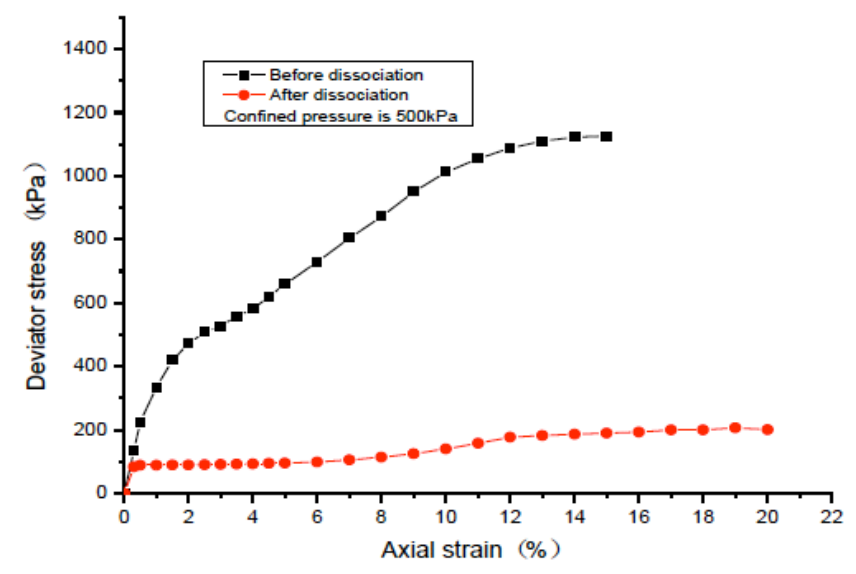

Fig. (8). Stress-strain curves before and after dissociation of GH.

to the saturated fine sand $[6,33,34]$. The results indicate that if the hydrate forms in a consolidated and cement skeleton, the dissociation affects mainly the cohesion, while the hydrate forms in an unconsolidated skeleton, the dissociation effects mainly the internal friction angle.

The mechanical parameters (such as elastic modulus and strength parameters) varied with the content of $\mathrm{MH}$, soil/rock skeleton's properties and experimental conditions although these parameters of bulk methane hydrate or ice or water are constants $[32,33,35-38]$ (Table 2). So it is necessary to present relations between these parameters and the content, distribution and mechanical properties of each phase (MH, soil/rock skeleton,gas, water).

\subsection{Thermal Properties}

The response of methane hydrate to changing thermal environments is controlled by its thermal conductivity, thermal diffusivity, and specific heat. These properties are described succinctly by Briaud and Chaouch [39]: "A high value of thermal conductivity means heat travels easily through the material; a high value of specific heat means that it takes a lot of heat to raise the temperature of the material; and a high value of diffusivity means that it will take little time for the temperature to rise in the material".

On localized length scales, thermal diffusivity and specific heat of methane hydrate are important not only for modeling controlled production of methane from hydrate $[40,41]$, but for hazard mitigation in conventional hydrocarbon extraction, which can destabilize hydrate underlying production equipment [39]. On local and regional scales, hydrate thermal conductivity is used in heat flow measurements [42]. Globally, hydrate thermal properties are needed to relate climate variability to methane released from hydrate [43].

Thermal Conductivity: Thermal conductivity is a key physical property for thermal modeling of gas hydratebearing sediment. Combined with local thermal gradients, thermal conductivity is used in constraining heat flow estimates. Combined with predictive models of hydrate dissemination and concentration, thermal conductivity is a critical parameter constraining the dynamic migration of hydrate dissociation fronts formed by localized heat input schemes for methane recovery [44]. The bulk thermal conductivity of HBS is a complex interplay among particle size, effective stress, porosity, and fluid-versus-hydrate filled pore spaces. The thermal conductivity of THF hydrate-bearing soils increases upon hydrate formation although the thermal conductivities of THF solution and THF hydrate are almost the same. Several mechanisms can contribute to this effect including cryogenic suction during hydrate crystal growth and the ensuing porosity reduction in the surrounding sediment, increased mean effective stress due to hydrate formation under zero lateral strain conditions, and decreased interface thermal impedance as grain-liquid interfaces are transformed into grain-hydrate interfaces [45]. The thermal conductivities of grains and fluids vary across several orders of magnitude: mineral grains (order of $10 \mathrm{~W} / \mathrm{K} 1$ ), water (order of 1 $\mathrm{W} / \mathrm{K} 1$ ), and air (order of $0.01 \mathrm{~W} / \mathrm{K} 1$ ). Such a contrast in thermal conductivities leads to preferential particle-level heat transport processes in granular materials: conduction along the mineral, particle-to-particle conduction across contacts, particle-fluid-particle conduction across the fluid near contacts, and conduction along the pore fluid within the pore space. Thermal conductivity decreases with increasing temperature and increases with increasing methane pore pressure. Despite the high thermal conductivity of quatz relative to methane hydrate, the largest thermal conductivity is in the mixture containing 33\% hydrate rather than in hydrate-free sand. This suggests that gas hydrate enhances grain-to-grain heat transfer, due to intergranular contact growth during hy- 
Table 2. Elastic Constants of Selected Sediment Components (After Literature [16])

\begin{tabular}{|c|c|c|c|c|c|}
\hline Material & $V_{p}(k m / s)$ & $\mathbf{V}_{\mathrm{s}}(\mathrm{km} / \mathrm{s})$ & K (GPa) & G (GPa) & $r\left(\mathrm{~kg} / \mathrm{m}^{3}\right)$ \\
\hline Methane gas (10 MPa, $273 \mathrm{~K})$ & 0.412 & 0 & 0.01 & 0 & 90 \\
\hline Water & 1.5 & 0 & 2250 & 0 & 1000 \\
\hline Ice Ih (5 MPa, $273 \mathrm{~K})$ & 3.87 & 1.94 & 9.09 & 3.46 & 917 \\
\hline Methane hydrate (5 MPa, $273 \mathrm{~K}$ ) & 3.77 & 1.96 & 8.41 & 3.54 & 925 \\
\hline HBS & $0.4 \sim 3^{\mathrm{a}}$ (Saturation $0 \sim 30 \%$ ) & $0.3 \sim 1.5^{\mathrm{a}}$ (Saturation $\left.0 \sim 30 \%\right)$ & & $0.096 \sim 0.128^{\mathrm{b}}$ & \\
\hline Clay & 3.41 & 1.63 & 20.9 & 6.85 & 2580 \\
\hline Quartz & 6.04 & 4.12 & 36.6 & 45 & 2560 \\
\hline
\end{tabular}

${ }^{\mathrm{a}}$ Priest et al., 2005; ${ }^{\mathrm{b}}$ Ogisako et al., 2007

drate formation. [46]. The thermal conductivity of sediments is proportional to the volumetric fraction of quartz. Interparticle conductive heat transfer is proportional to the particle radius and inversely proportional to the intercontact distance. The higher the packing density and coordination number, the higher the thermal conductivity of the sediment. Water acts as a relatively high conductivity bridge between particles. Therefore, the thermal conductivity of partially saturated soils increases with the degree of saturation. Increasing effective stress leads to higher thermal conductivity [47].

As shown in Table, the thermal conductivities of methane hydrate and water differ by $<10 \%$ at the temperatures found in hydrate-bearing sediments [48, 49]. For this reason, firstorder thermal conductivity estimates can neglect the presence of methane hydrate and assume the sediment pore space contains only water [50].

Specific Heat: Specific heat, a measure of the heat stored in the system, is a controlling parameter during hydrate dissociation. Hydrate dissociation is an endothermic process, and the dissociation rate can be limited if insufficient heat is available at the dissociation front. The specific heat of methane hydrate is less than half that of water, meaning the presence of hydrate can significantly lower the specific heat of hydrate-bearing sediment relative to water-saturated sediment [49].

Unlike thermal conductivity, specific heat depends only on the mass fractions of sediment, hydrate, and water rather than on their pore-scale distribution and interfacial effects. Using the subscripts $\mathrm{m}, \mathrm{w}$, and $\mathrm{h}$ to refer to the host sediment mineral, pore water, and methane hydrate, respectively, the formation's bulk specific heat, c.p, is given for a gas-free system by the weighted average of the mass fractions of the sediment. Because the specific heat of methane hydrate is less than half that of water, hydrate formation can significantly lower the specific heat of hydrate-bearing sediments [49].

Thermal Diffusivity: Thermal diffusivity is important when describing how a thermal front moves through a system. For an example, when high-temperature hydrocarbons are drawn up through a well to the seafloor, temperature increases in sediment and the time required to raise the sediment temperature to a given value is proportional to $1 / \mathrm{k}_{\text {eff }}$, where $k_{\text {eff }}$ is the effective thermal diffusivity [6, 39]. The thermal diffusivity of methane hydrate is more than twice that of water; therefore, hydrate-bearing sediments can change temperature more rapidly than hydrate free sediments [49]. In sediment with porosity $\varepsilon=35 \%$, a hydrate saturation $\mathrm{S}_{\mathrm{h}}=35 \%$ increases heating rates by more than $10 \%$ relative to those in the absence of hydrate. Thermal diffusivity can be calculated by the formula $\mathrm{k}=\lambda / \rho / \mathrm{c}_{\mathrm{p}}$ without considering the mixing effects of HBS, where $\lambda$ is the thermal conductivity, $\rho$ the density, $c_{p}$ the specific heat.

Enthalpy of Reaction: The organized hydrate structure has less internal energy than a freely moving, disordered combination of methane and water, so energy must be released for hydrate to form and reabsorbed for hydrate to dissociate [51]. This energy change is defined as the enthalpy of reaction or latent heat $(\Delta \mathrm{H})$. According to Sloan [1] to a first approximation the dissociation enthalpy is a function of the following factors: the hydrogen bonds making up the lattice, the cavity occupation, and the guest size. About $80 \%$ of the total dissociation enthalpy is due to the strength of the water hydrogen bonds. The dissociation enthalpy is defined as the enthalpy change to decompose the gas hydrate. Two types of gas hydrate dissociation are generally distinguished: the decomposition of gas hydrates into ice and gas, and the decomposition into gas and liquid. The difference in enthalpy between both reactions is assumed to be equal to the enthalpy of fusion of ice. Calorimetry can provide measurements of $\Delta \mathrm{H}$, but only a limited number of studies are available. Enthalpies can also be estimated from phase equilibrium and thermodynamic data using the Clausius-Clapeyron equation to relate pressure, $\mathrm{P}$; temperature, $\mathrm{T}$; enthalpy, $\Delta \mathrm{H}$; and compressibility,

$$
\frac{d \ln P}{d(1 / T)}=\frac{-\Delta H}{Z R}
$$

where $\mathrm{R}$ is the ideal gas constant. The validity of this method is contingent upon negligible changes in compressibility. $\Delta \mathrm{H}$ depends on the guest molecule but is primarily controlled by the number of hydrogen-bonded water molecules. Typically, there are 6 water molecules per guest molecule in structure I hydrate but 17 per guest in structure II hydrate [1,52].

For modeling temperature profiles, thermal diffusivity is of paramount importance. Determine the thermal parameters (thermal diffusivity, conductivity et al.) is complicated be- 
Table 3. Thermal Properties of HBS (Referenced from Literature [16])

\begin{tabular}{|c|c|c|c|c|}
\hline Material & $\lambda\left(\mathbf{W} \mathbf{m}^{-1} \mathbf{K}^{-1}\right)$ & $\kappa\left(m^{2} s^{-1}\right)$ & $\mathrm{C}_{\mathrm{p}}\left(\mathrm{J} \mathrm{kg}^{-1} \mathrm{~K}^{-1}\right)$ & $\rho\left(\mathbf{k g ~ m}^{-3}\right)$ \\
\hline Air & 0.024 & $183 \times 10^{-7}$ & $1010(273 \mathrm{~K})$ & $1.298(272 \mathrm{~K})$ \\
\hline Water & $0.58(283 \mathrm{~K})$ & $1.38 \times 10^{-7}$ & $4192(283 K)$ & $999.7(283 \mathrm{~K})$ \\
\hline Ice & $2.21(270 \mathrm{~K})$ & $11.7 \times 10^{-7}$ & $2052(270 \mathrm{~K})$ & $917(273 \mathrm{~K})$ \\
\hline Methane gas & $0.0297(260 \mathrm{~K}, 1 \mathrm{MPa})$ & $18.0 \times 10^{-7}$ & $2170(260 \mathrm{~K})$ & $7.61(260 \mathrm{~K}, 1 \mathrm{MPa})$ \\
\hline Methane hydrate & $0.57(263 \mathrm{~K})$ & $3.35 \times 10^{-7}$ & $2031(263 K)$ & $929(263 \mathrm{~K})$ \\
\hline $\begin{array}{l}\mathrm{THF}+\text { water } \\
\text { THF. } 17 \mathrm{H}_{2} \mathrm{O}\end{array}$ & $0.47(283 \mathrm{~K})$ & $3.12 \times 10^{-7}$ & $4080(282 \mathrm{~K})$ & $982(283 \mathrm{~K})$ \\
\hline $\begin{array}{l}\text { THF hydrate } \\
\text { THF. } 17 \mathrm{H}_{2} \mathrm{O}\end{array}$ & $0.5(261 \mathrm{~K})$ & $2.55 \times 10^{-7}$ & $2020(261 \mathrm{~K})$ & $971(273 \mathrm{~K})$ \\
\hline Quartz & 7.7 to 8.4 & $41 \times 10^{-7}$ & $730(273 \mathrm{~K}) 2650$ & \\
\hline
\end{tabular}

cause it requires quantifying heat transfer while the hydration reactions generate heat as the specimen changes gradually from a liquid to a solid [53]. However, the thermal property data for well-characterized mixtures of sediment and pure gas hydrate are lack. A few studies provided qualitative insight into the dependence of thermal properties on methane hydrate formation in methane gas + water as well as methane gas + water + sediment systems. Relative volumes and distributions of individual phases in the samples were poorly known, making it difficult to apply these results to other hydrate-bearing systems. To avoid these limitations, some special sampling and measurement techniques, such as IceSeeding Method and needle probe method [46,53], are adopted. Some thermal properties of air, water, ice, methane gas, methane hydrate and THF + water are shown in Table 3.

\subsection{Permeability}

The permeability of HBS has a great influence on the process of dissociation and formation of $\mathrm{GH}$ and decreases gradually with the formation of $\mathrm{GH}$ in pores, which causes the gas percolation decrease or even clogged. Generally, hydrate accumulation and permeability clogging occur at a faster rate in the porous medium than in the fractured system. So inversely, the decrease of permeability lowers or even stops the formation of GH [54-56]. By reducing the pore size and changing the pore shape. Pore-filling hydrate reduces the permeability more significantly than mineralcoating hydrate [57]. Based on the limited data available for gas free, hydrate-bearing systems [58], pore-filling hydrate models provide the best estimates of permeability $[59,60]$.

Hydrate accumulation in the pore space of a porous medium reduces absolute permeability to water or gas, and depending on the specific hydrate location within pore spaces, the impact of gas hydrate saturation on the permeability and relative permeability (which accounts for the presence of a second mobile phase in the pore space) can vary significantly. There is a significant volume of smaller pores or immobile regions that do not significantly contribute to permeability if the pores in the sediment are broadly distributed. The reason is that the hydrate blocks pores and causes dead end pores, which leads to the non-uniformity of permeabil- ity. Generally, the effective absolute permeability does not decrease significantly until hydrate saturation reaches $\sim 40 \%$. Beyond that point the permeability decreases rapidly as hydrate saturation increases, and becomes nearly zero before the pore space is fully filled with hydrate [61].

At present, Darcy law is widely used in the analysis of the production of gas hydrate from sediment though it is not practical when the sizes of the pores and throats are smaller than some value. In fact, the permeability of HBS changes with the formation or dissociation of GH. For simplicity, the permeability is mostly assumed as the function of the porosity and the specific surface area, for an example, the model presented by [62]:

$$
k=\frac{\phi^{3}}{2 A_{s}^{2}}
$$

in which $k$ is the absolute permeability, $\phi$ is the porosity, $A_{s}$ is the specific surface area.

The permeabilities of HBS are affected by fractures, pores, throats, laminae, and veins [63]. Thus micro measurement is often adopted, for an example, by using proton nuclear magnetic resonance (NMR), X-ray computerized tomography (CT) to image and nondestructive measure the density and microstructure for evaluating the permeability $[58,64]$.

In most sedimentary systems including HBS, fluid transport occurs through a heterogenerous permeability network that can be represented as a combination of fractures and a porous medium. So microscopically, the permeability of the medium is controlled by the degree of connectivity of pore space, pore size distribution, fracture width and length. The conventional approach to study the impact of geological uncertainty on reservoir recovery is to build a detailed reservoir model using geophysical and geological data, and then perform flow simulation [65-67]. If fractures are poorly interconnected and the matrix rock is relatively impermeable, the flow may be blocked in the network of fractures. Otherwise, if the matrix is permeable and the fractures are regular and highly interconnected, fractures and matrix can be treated as 
separate continuums occupying the entire domain. In order to estimate the performance parameters, it is necessary to construct reservoir models (with associated probability) and run flow simulations. This method is time costing and computationally expensive. Therefore, there is a great incentive to produce much simpler physically-based models to quickly predict uncertainty in performance.

Percolation theory, especially dual percolation theory [68-71] and pore network simulation [72-75] are effective methods to investigate the connectivity of reservoir. Dual percolation model is constituted of fracture network and matrix percolation network. Each fracture's center is mapped as a "site" of matrix porous network, and the "bond" is mapped as the connection between porosity. Fracture network is used to model the flow in fractures while matrix network is used to model the flow in porosity and defects. The coupling of these two types of models can simulate the formation of interporous flow channel. The permeability of fractural porous media can be presented based on the connective degree to obtain [76].

The presence of gas hydrate in the sediment dramatically influences the flow of water and gas through that sediment. However, very few permeability and relative permeability measurements of HBS have been performed. More investigated work is required to clarify how gas hydrate occupies the pore space in natural porous media and in laboratory samples and how this affects transport parameters. For this target, some imaging techniques such as X-ray computed tomography (CT), nuclear magnetic resonance (NMR) should be used in measurements $[59,77]$.

\section{MECHANICS OBTAINED FROM IN-SITU INVES- TIGATIONS}

\subsection{Seismic Geophysical Method and Measured Results}

Compressional $\mathrm{P}$ waves and shear $\mathrm{S}$ waves are extensively used for mapping hydrate occurrences and estimating the hydrate saturation within those occurrences. Acoustic remote sensing is possible because the presence of hydrate stiffens the host sediment, increasing the $\mathrm{P}$ and $\mathrm{S}$ wave velocities. Seismic geophysical methods mainly include singlechannel seismic method, multi-channel seismic method, multi-frequency method and multi-component method. The parameters of HBS such as elastic modulus, damping and contents are obtained by the inversion according to the wave speed and the attenuation characteristics [78]. Some researchers $[79,80]$ applied the multi-attribute analysis to measure the HBS parameters such as porosity, density etc. in Blake Ridge. It was proved that it was a practical method to obtain the two-dimensional data of HBS. Rajput et al. [81] applied the AVO (amplitude versus offset) technique in determining the properties of HBS.

Whiffen et al. [82] used the technique of Brillouin spectroscopy to determine the longitudinal sound velocities in pure, artificially grown, polycrystalline samples of methane hydrate and xenon hydrate. For methane and xenon, the relative sound velocity of hydrate to ice was 0.88 and 0.76 , respectively. Bathe et al. [83] presented measured ultrasonic wave velocities in structure II polycrystalline THF hydrate.
At $-17^{\circ} \mathrm{C}$ the measured longitudinal wave velocity was 3513 $\mathrm{m} / \mathrm{s}$, and the shear wave velocity was $1663 \mathrm{~m} / \mathrm{s}$. The hydrate values were somewhat less than the corresponding values for multigranular ice at $-5^{\circ} \mathrm{C}$. Kiefie et al. [84] used Brillouin spectroscopy to determine acoustic velocities of eight different hydrates, including methane and R11 (Freon-11). The experimentally determined longitudinal velocity ratio of hydrate to that of ice was 0.88 (similar to the result of Whiffen et al. [82]) for methane hydrate and 0.91 for R11 hydrate. The ratio for THF hydrate, 0.96 , is considerably higher. THF is a commonly used low-pressure hydrate former and miscible with water, while R11 is not. Wittebolle and Sego (1985) [85] measured compressional wave velocities of sediments (quartz sand) containing gas hydrate formed from a Freon-12 gas source. A P wave velocity of $3600 \mathrm{~m} / \mathrm{s}$ was measured at $1.7^{\circ} \mathrm{C}$ and dropped to $2500 \mathrm{~m} / \mathrm{s}$ at $+1.9^{\circ} \mathrm{C}$. The $\mathrm{P}$ wave velocity was $1900 \mathrm{~m} / \mathrm{s}$ with no hydrate present. Pearson et al. [86] reported an increase in sonic velocities and electrical resistivity in hydrate-bearing sediments as compared to sediments without hydrate.

The maximum thickness of HBS in nature is of the hectometer size, while the minimum thickness is less than $1 \mathrm{~m}$. The matrix of HBS can be consisted of sand, clay, silty sand et al. [87] which have different characteristics of wave propagation and reflection. Thus the thickness of HBS obtained by conventional seismic wave methods has relatively large error because of the low resolution. It can be overcome by utilizing high resolution seismic wave methods. Up to now, there are no general models to describe the relations between mechanical properties and wave speeds which is the basis of wave inversion. Thus the seismic wave inversion technique for HBS is still a difficult problem [88, 89].

Generally, the mechanical properties obtained by geophysical seismic methods are average and rough in large scale. Other methods such as coring method, logging method etc must be used to obtain more accurate data in small scale.

\subsection{Method of Drilling Core and Measured Results}

Method of drilling core is to obtain the mechanical and physical properties of HBS by sampling and testing [90, 91]. This method is widely used in the ODP [92]. The test methods used to measure the contents of carbon-hydrogen and other components include $\chi$-ray diffraction analysis, color reflection spectrophotometry analysis, biostratigraphy analysis, calcium microfossil analysis, pore water geochemical analysis etc. MST (multisensor track) method can be used to measure the thermal conductivity, humidity, density, strength.

Natural gas can exist in the form of solid, liquid and gas in deep ocean beds relying on the in-situ temperature and pressure. It is dissolved if its concentration in the pore water is lower than the solubility. The excess gas exists in the form of gas. The concentration and the existing form of natural gas in deep ocean beds are important data to understand the formation kinetics of GH in sediment and the physical properties of HBS.

The formation of GH strengthens the sediment, while the dissociation is opposite due to the damage of the soil matrix 
and the decrease of the effective stress by the produced excess pore water pressure and gas pressure. The properties of HBS obtained in laboratory can not be the same as that insitu if GH dissociates during sampling and being seated in test apparatus since GH are very sensitive to the temperature and pressure. So it is very important that keep HBS cores under the same conditions as that in-situ before and during doing tests for obtaining practical mechanical properties.

In a word, one of the key problems by drilling core method is to maintain the temperature and pressure during sampling and moving from the container to the test apparatus [37].

\subsection{Geophysical Well-Logging Method and Measured Results}

Geophysical well-logging method for obtaining the physical and mechanical properties of HBS in a well profile is another effective method except for the geophysical seismic method and the drilling core method. This method is mature in the petroleum exploration, so some experiences and techniques can be perfect references for $\mathrm{GH}$ exploration. The geophysical well-logging methods include caliper logging, gamma-ray, spontaneous potential, resistivity, sonic wave and neutron porosity-measurement method etc. [21, 93]. The physical and mechanical properties of HBS are different from other media (saturated soil, oil stratum etc.), for an example, the existence of $\mathrm{GH}$ causes the increase of wave velocity, resistance, caliper and the decrease of the density. The resistance method and the sonic wave method are shown to be the most useful in practices. However, Geophysical well-logging method is not effective for the measurement of silty sand with low saturation degree of $\mathrm{GH}$ and argillaceous $\mathrm{GH}$ because the detection range is limited by the wave length and energy.

\section{THE THEORETICAL MODELS FOR HBS'S ME- CHANICAL PARAMETERS}

\subsection{Main Theoretical Models}

The attenuation of seismic waves within hydrate-bearing soils is sensitive to the volume of gas hydrate within the pore space. An attenuation peak was observed when the percentage of hydrate within the pore space was between 3 and 5 percent. The peak in attenuation is thought to be due to cementation of grain contacts leading to an increase in low aspect ratio cracks between the hydrate and sand grains. This increase in the effective area of the grain contacts led to increased dissipation of mechanical wave energy due to the flow of adsorbed water (and or free gas) along the crack into the open pore space, and then back again during the passage of a seismic wave. Attenuation measurements on dissociated specimens do not show any appreciable attenuation peak and only a small increase in attenuation from the air dry specimens due to the increase in water at grain contacts. Once the grain contacts were saturated, no appreciable change was noted with increasing water saturation [94, 95].

The acoustic properties of HBS showed dramatic changes with the saturation degree of GH. The wave velocity and attenuation are two important parameters by which the mechanical properties of HBS can be determined [96, 97]. Gei and Carcione's [98] showed that the wave velocity and the mechanical properties of HBS are mainly the functions of the pore pressure, temperature and saturation degree.

The saturation of gas hydrate and ice, sediment types, hydrate formation mechanism all affect the measured acoustic properties and the strength of HBS. Generally, Vp ranges from less than $1 \mathrm{~km} / \mathrm{s}$ for gas-charged sediment; to $1.77-1.94$ $\mathrm{km} / \mathrm{s}$ for water-saturated sediment; $2.91-4.00 \mathrm{~km} / \mathrm{s}$ for sediment with varying degrees of hydrate saturation; and 3.88 $4.33 \mathrm{~km} / \mathrm{s}$ for frozen sediment. Vp measured in fine-grained sediment containing gas hydrate is substantially lower (1.97 $\mathrm{km} / \mathrm{s}$ ) [26].

To obtain the contents and mechanical properties of HBS by inversion analysis according to the data from geophysical survey such as the wave speed and attenuation characteristics, the relations between the wave speed, the attenuation characteristics and the contents of GH and mechanical parameters $[95,99]$ must be determined first. The theoretical relations are divided into two aspects: (1) the relations between the content and the wave speed of each phase and the wave speed of HBS. (2) the relations between the wave speed of HBS and the mechanical parameters such as elastic modulus, shear modulus. Based on these two kinds of relations, on one hand, the mechanical parameters of HBS and the content of each phase can be determined by inversion analysis by the wave speed of HBS. On the other hand, the mechanical parameters of HBS can be determined by the mechanical parameters and content of each phase.

As described in the above, there are three existing forms of $\mathrm{GH}$ in HBS: firstly, $\mathrm{GH}$ is a part of pore fluid, secondly, $\mathrm{GH}$ is a part of soil matrix, thirdly, $\mathrm{GH}$ cements the grains. Different existing forms have different influences on the mechanical properties of HBS. Kinds of theoretical models describing HBS's mechanical parameters are presented based on the three existing forms. The main models include time-average model $[100,101]$, cementation model [102104], pore-filling model [105], weight-average model [106109], effective medium model [103, 110].

In the time-average model the whole sediment slowness (inverse of the velocity) is taken as the weighted sum of the various constituents. However, the effects of the cementation of hydrate are not considered in this model. Pearson [106] presented the weight-average model considering the characteristics of pores full filled with fluid and under-consolidated $\mathrm{GH}$ near the ocean-floor. The velocity of HBS in this model must be the weight-average of the speeds obtained by kinds of methods. The main formula is as follows

$\frac{1}{V_{p}}=\frac{\phi(1-S)}{V_{w}}+\frac{\phi S}{V_{h}} \frac{1-(1-S)}{V_{m}}$

in which $V_{p}, V_{w}, V_{h}$ and $V_{n}$ are the compressive wave speed of HBS, pore liquid, GH and matrix respectively, $\phi$ the porosity, $S$ concentration of GH in the pore space. Eq. (3) is only suit for the case in low speed of skeleton, e.g. the condition of no consolidation [111].

Lee et al. [107]) combined the time-average method with the wood formula [112] by using of the Nobes' method [113] based on the Pearson's weighting method [106], presented the following weighted-average equation 


$$
\frac{1}{V_{p}}=\frac{W \phi(1-S)^{n}}{V_{p 1}}+\frac{1-W \phi(1-S)^{n}}{V_{p 2}}
$$

in which $V_{p}$ is the compressive wave speed of HBS, $V_{p 2}, V_{p 2}$ the compressive wave speed of HBS determined by the Wood method and the time-average method respectively, W the weighted factor, $n$ a constant indicating the lithifaction velocity.

Weighted-equation can forecast well the elastic properties of HBS containing gas, but this equation is empirical because of the adoption of the empirical relation of timeaverage formula.

Lee et al. $[108,109]$ presented a method to forecast the elastic wave speed of under-consolidated HBS, based on the Biot's [114, 115] and Gassmann's theories [116], assuming that the ratio of shear wave speed to compressive wave speed is proportional to the speed ratio of skeleton to density. This formula has been used in the data logging and analysis in the Canadian mallik-2L well. The main equation is as follows

$V_{p}=\sqrt{\frac{k+\frac{4 \mu}{3}}{\rho}}, V_{s}=\sqrt{\frac{\mu}{\rho}}, \rho=(1-\phi) \rho_{m a}+\phi \rho_{f l}$

in which $k, \mu, \rho$ are volume modulus, shear modulus and density of mixture, respectively, $\rho_{m a}$ the density of skeleton, $\rho_{f l}$ the density of pore fluid.

Helgerud [103] and Jackobsen [110] improved the rate equation which is based on the effective medium theory as follows

$V_{p}=\sqrt{\frac{K_{s a t}+\frac{4 G_{\text {sat }}}{3}}{\rho_{B}}}, V_{s}=\sqrt{\frac{G_{s a t}}{\rho_{B}}}$

in which $\rho_{B}$ is the volume density, $K_{\text {sat }}$ the volume modulus, $G_{\text {sat }}$ the shear modulus and equaling to the shear modulus of dry sediment $G_{d r y}$, and

$$
K_{s a t}=K \frac{\phi K_{d r y}-\frac{(1+\phi) K_{f} K_{d r y}}{K}+K_{f}}{(1-\phi) K_{f}+\phi K-\frac{K_{f} K_{d r y}}{K}}
$$

in which $\mathrm{K}$ is the volume modulus of skeleton, $K_{d r y}$ the volume modulus of dry sediments, $K_{f}$ the volume modulus of pore fluid.

The advantage of the model based on effective medium theory is that, similar to the weighted average models, it can effectively forecast the elastic properties of loose and gascontaining HBS, while the disadvantage is that the shear wave speed of high-porosity sediment forecasted by this model is unreasonable large.

A linear expression similar to the weighted average of the three-phase (ice, hydrate, soil matrix) is presented by Zhang et al. [32] assuming it is the combination of porefilling type and grain-adhering type, the expression is: $\varepsilon_{t} E=a \varepsilon_{H} E_{H}+b \varepsilon_{I} E_{I}+c \varepsilon_{s} E_{s}+d \frac{\varepsilon_{H}}{E_{H}}+e \frac{\varepsilon_{I}}{E_{I}}+f \frac{\varepsilon_{s}}{E_{s}}$

in which $E_{H}, E_{r}, E_{I}$ are the elastic modulus of hydrate, soil skeleton, and ice respectively, $E$ the elastic modulus of HBS, $\varepsilon_{H}, \varepsilon_{r}, \varepsilon_{I}$ saturation of hydrate, soil matrix and ice, $\varepsilon_{t}=\varepsilon_{H}+\varepsilon_{I}+\varepsilon_{s} a \sim f$ constant coefficients .

At present, there are not enough data on the mechanical properties of HBS and each phase, thus the relations between the property of each phase and the mechanical parameters (e.g. shear modulus, Yang's modulus and Poisson's ratio) is hypothetical which needs to prove further. In theoretical models on elastic constants and wave characteristics, the cementation between GH and skeleton, effects on the microstructure of HBS caused by the formation of GH should be considered. The relationship between GH content, GH distribution, microstructure characteristics, chemical characteristics, biological characteristics and elastic constants and wave properties of HBS should be especially established, so as to provide a foundation for elaborate exploration of $\mathrm{GH}$.

\subsection{Application and Prospect}

Geophysical methods are generally used to estimate the existence and the mechanical properties of HBS in a large scale $(\sim 1000 \mathrm{~m})$, based on the inverse analysis of the seismic data. Drilling core methods are used to determine the physical and mechanical properties of HBS in a smaller scale $(\sim 1 \mathrm{~m})$ than that of Geophysical methods.

Many measures on the mechanical properties of HBS had been processed at the Canadian Mallik permafrost and at Blake Ridge (ODP Leg 164) [24] to estimate the existence of GH in the Arctic Circle and to obtain physical and mechanical properties of HBS [117].

The present technique can hardly maintain the in-situ conditions of high pressure and low temperature during coring and before testing, so it is difficult to do mechanical tests without dissociation of GH in samples. Meanwhile, dissociation of GH can cause cores fracture or burst. Therefore, some routine methods of geological survey can not be used in the exploration of HBS, in other words, new equipments and methods are required, such as the pizo-cone penetration test (CPTU) which is widely used in ocean geological investigations because of its well repeatability, mature technique and less disturbance to the sediment [118].

\section{CONCLUSIONS}

The formation course of HBS, the properties of skeleton, the content and distribution of gas in HBS, microbe, the content of calcium microfossil is closely related with the mechanical properties of HBS.

Hydrate saturation has small effects on the shear strength of HBS when the saturation is less than about $30 \%$ and high when the saturation is larger than about $40 \%$. The strength and stiffness of HBS depend strongly on the hydrate formation history. For an example, the strength will pronouncedly increase even if there is only a small amount of hydrate 
forms if the hydrate cements the particles. In contrast, there is a measurable effect on theses parameters only when the hydrate saturation exceeds about $40 \%$ if the hydrate is only filled in the pore without cementation with particles. However, the internal friction angle is nearly independent of hydrate saturation.

The mechanical parameters (such as elastic modulus and strength parameters) varied with the content of $\mathrm{MH}$, soil/rock skeleton's properties and experimental conditions although the parameters of bulk methane hydrate or ice or water are constants.

Particle and pore-scale interactions between hydrate and its host sediment lead to a richly complex system of interdependent macroscale physical properties that govern the evolution of hydrate-bearing sediments. Understanding these interdependencies can provide a framework for understanding the hydrate-bearing sediments as well as providing a robust basis for preliminary evaluations of gas production strategies and instability conditions.

The host sediment's silt and clay content determine the mode of hydrate occurrence. In coarse grained systems with fine content below $7 \%$, hydrate generally occurs in the pore space between grains. When the fine content exceeds $15 \%$, hydrate is found disseminated in the sediment and forming veins and nodules that displace sediment grains.

The study in the future should be aimed at: (1) in the field in-situ exploration, more elaborate method such as CPTU should be adopted. (2) Particle and pore-scale interactions between hydrate and its host sediment should be clarified further. (3) The effects of the amount of fines, their mineralogy, and specific surface area on the mechanical and thermal properties of hydrate-bearing sediments should be further investigated. (4) Routinely image specimens to visualize hydrate distribution so that proper data inversion procedures can be implemented in the interpretation of measured properties. (5) Relations between mechanical and thermal properties and the physical characteristics such as porosity, fractions of components, mineral types should be built. (6) Practical relations between strain and stress considering the physical characteristics should be presented.

\section{CONFLICT OF INTEREST}

The authors confirm that this article content has no conflicts of interest.

\section{ACKNOWLEDGEMENT}

This paper is supported by The National Natural Science Foundation of China (No.11272314; No. 51239010).

\section{REFERENCES}

[1] E.D. Sloan, Clathrate hydrates of natural gases, Marcel Dekker Inc.: New York, 1998.

[2] J. L. Cox, Natural gas hydrates: properties, occurrence and recovery, Bosten: Butterworth, 1988.

[3] K.A. Kvenvolden and T.D. Lorenson, "The global occurrence of natural gas hydrate", Geophys. Monogr., vol.124, pp.3-18, 2001.

[4] H.O. Kono, S. Narasimhan, F. Song, and D. H. Smith, "Synthesis of methane gas hydrate in porous sediments and its dissociation by depressurizing", Powder Technol., vol.122, pp.239-246, 2002.
[5] A.M. Tréhu, G. Bohrmann, F.R. Rack, M.E. Torres, L. H. Nancy and R. S. Kenneth, "Explanatory Notes", In: Proceedings of Ocean Drilling Program, Initial Reports, 2003, p. 204.

[6] X.H. Zhang, X.B. Lu and Q.P. Li, "Thermally induced evolution of phase transformations in gas hydrate sediment", Sci. China Phys. Mech. Astron., vol.53, no. 8, pp.1530-1535, 2010a

[7] X. B. Lu, S. Y. Wang, X. H. Zhang, Q. P. Li and X. H. Zeng, "A mathematical model for dissociation of gas hydrate", In: Proceedings of the $19^{\text {th }}$ International Offshore and Polar Engineering Conference, Ocean Mining Symposium, 2009, pp.202-206.

[8] T. J. Kneafsey, L.Tomutsa, G. J. Moridis, Y. Seol, B. M. Freifeld, C. E. Taylor and A. Gupta, "Methane hydrate formation and dissociation in a partially saturated core-scale sand sample", J. Petrol. Sci. Eng., vol. 56, no.1-3, pp.108-126, 2007.

[9] E. Spangenberg, J. Kulenkampff, R. Naumann, and J. Erainger, "Pore space hydrate formation in a glass bead sample from methane dissolved in water", Geophys. Res. Lett., vol. 32, no. 24, PP. L24301, 2005.

[10] Y. Zhong, and R. E. Rogers, "Surfactant effects on gas hydrate formation", Chem. Eng. Sci., vol. 55, no. 19, pp.4175-4187, 2000

[11] W. F. Waite, W. J. Winters and D. H. Mason, "Methane hydrate formation in partially water-saturated Ottawa sand", Am. Mineral., vol. 89, no.8-9, pp.1202-1207, 2004.

[12] A. Klapproth, K. S. Techmer, S. A. Klapp, M. M. Murshed, and W. F. Kuhs, "Microstructuple of gas hydrates in porous media", In: the $11^{\text {th }}$ International Conference of Physical and Chemical Ice, 2007, pp. 321-328.

[13] L. A. Stern, S. H. Kirby and W. B. Durham, "Peculiarities of methane clathrate hydrate formation and solid-state deformation, including possible superheating of water ice", Science, vol. 273, no.5283, pp.1843-1848, 1996.

[14] L.A. Stern, S.H. Kirby and W.B. Durham, "Polycrystalline methane hydrate: Synthesis from superheated ice, and low-temperature mechanical properties", Energy Fuels, vol. 12, no. 2, pp.201-211, 1998.

[15] T.S.Yun, J.C. Santamarina and C. Ruppel, "Mechanical properties of sand, silt, and clay containing tetrahydrofuran hydrate", J. Geophys. Res., vol. 112, pp. B04106, 2007.

[16] W.F.Waite, J.C. Santamarina, D.D. Corts, B. Dugan, D. N. Espinoza, J. Germaine, J. Jang, J. W. Jung, T. J. Kleafsey, H. Shin, K. Soga, W. J. Winters, and T. S. Yun, "Physical properties of hydrate-bearing sediments", Rev. Geophys., vol. 47, pp. RG4003, 2009.

[17] M.B.Helgerud, J. Dvorkin, A. Nur, A. Sakai, and T. Collett, "Elastic-wave velocity in marine sediments with gas hydrate: effective medium modeling", Geophys. Res. Lett., vol.26, no.13, pp.20212024,1999.

[18] J. Dvorkin, J. Berryman and A. Nur, "Elastic moduli of cemented sphere packs", Mech. Mater., vol. 31, no. 7, pp.461-469, 1999.

[19] C.R.I. Clayton, J.A. Priest, and A.I. Best, "The effects of dissemininated methane hydrate on the dynamic stiffness and damping of a sand", Geotechnique, vol. 55, no. 6, pp.423-434, 2005.

[20] B. D. William, H. K. Stephen, L. A. Stern and W. Zhang, "The strength and rheology of methane clathrate hydrate", J. Geophys. Res., vol.108, no.B4, pp. 2-1-2-11, 2003.

[21] F. Francisca, T.S.Yun, C. Ruppel, and J. C. Santamarina, "Geophysical and geotechnical properties of near sea-floor sediments in the northern gulf of Mexico gas hydrate province", Earth Planet. Sci. Lett., vol. 237, no. 3-4, pp.924-939, 2005.

[22] W.B. Durham, S.H. Kirby and W. Zhang, "The strength and rheology of methane clathrate hydrate", J. Geophys. Res., vol.108, no.B4, pp.2182, 2003.

[23] M. Hyodo, Y. Nakata, N. Yoshimoto, and R. Orense, "Shear behaviour of methane hydrate-bearing sand", In: Proceedings of the $7^{\text {th }}$ International Offshore and Polar Engineering Conference, Ocean Mining Symposium, 2007, pp.1326-1333.

[24] W.J. Winters, S.R. Dallimore, T.S. Collett, K. A. Jenner, J. T. Katsube, R. E. Cranston, J. F. Wright, F. M. Nixon, and T. Uchida, "Relation between gas hydrate and physical properties at the Mallik 2L-38 research well in the Mackenzie Delta", Ann. N. Y. Acad. Sci., pp.94-100, 2000.

[25] W. J. Winters, I.A. Pecher, W.F. Waite, and D. H. Mason, "Physical properties and rock physical models of sediment containing natural and laboratory-formed methane gas hydrate", Am. Mineral., vol. 89, no. 8-9, pp.1221-1227, 2004. 
[26] W.J. Winters, W.F. Waite, D.H. Mason, L. Y. Gilbert and I.A. Pecher, "Methane gas hydrate effect on sediment acoustic and strength properties", J. Petrol. Sci. Eng., vol.56,no.1-3 pp.127-135, 2007.

[27] A. Masui, H. Haneda, Y. Ogata and K. Aoki, "Effect of methane hydrate formation on shear strength of synthetic methane hydrate sediment", In: Proceedings of the $15^{\text {th }}$ International Offshore and Polar Engineering Conference, 2005, pp. 364-369.

[28] Masui, H. Haneda, Y. Ogata and K. Aoki, "Mechanical properties of sandy sediment containing marine gas hydrates in deep sea offshore Japan", In: Proceedings of the $7^{\text {th }}$ International Offshore and Polar Engineering Conference, Ocean Mining Symposium, 2007, pp.53-56.

[29] E. Ogisako, S. Nishio and A. Denda, "Simulation of triaxial compression tests on soil samples obtained from seabed ground in depp sea by elasto-viscoplastic constitutive equation", In: Proceedings of the $7^{\text {th }}$ International Offshore and Polar Engineering Conference, Ocean Mining Symposium, 2007, pp. 63-68.

[30] K. Miyazaki, A. Masui, H. Haneda, Y. Ogata, K. Aoki and T. Yamaguchi, "Variable-compliance-type constitutive model for Toyoura sand containing methane hydrate", In: Proceedings of the $7^{\text {th }}$ International Offshore and Polar Engineering Conference, Ocean Mining Symposium, 2007, pp 57-62.

[31] K. Miyazaki, T. Yamaguchi, Y. Sakamoto, N. Tenma, Y. Ogata and K. Aoki, , "Effects of confining pressure on mechanical properties of artificial methane hydrate bearing sediments", In: Proceedings of the $20^{\text {th }}$ International Offshore and Polar Engineering Conference, 2010, pp.132-137.

[32] X. H. Zhang, S. Y. Wang and Q.P. Li, "Experimental study of mechanical properties of gas hydrate deposits", Rock Soil Mech., vol. 31, no. 10, pp.3069-3074, 2010b, (in Chinese).

[33] X. B. Lu, L. Wang, S. Y. Wang and Li Q. P., "Study on the mechanical properties of Tetrahydrofuran hydrate deposit", In: Proceedings of the $18^{\text {th }}$ International Offshore and Polar Engineering Conference, 2008, pp. 57-60.

[34] X. H. Zhang, X. B. Lu, S. Y. Wang and Q. P. Li, "Experimental study of static and dynamic properties of tetrahydrofuran hydratebearing sediment", Rock Soil Mech., vol. 32, no. Supp. 1, pp.303308, 2011, (in Chinese).

[35] L.I. Berge, K.A.Jacobsen and A.Solstad, "Measured acoustic wave velocities of Rll (CCI3F) hydrate samples with and without sand as a function of hydrate concentration", J. Geophys. Res., vol.104, no.B7, pp.15415-15424, 1999.

[36] Y.H. Li, Y.C. Song, F. Yu, W. G. Liu and J. F. Zhao, "Experimental study on mechanical properties of gas hydrate-bearing sediments using Kaolin clay", China Ocean Eng., vol.25, no. 1, pp.113 $-122,2011$.

[37] Y.C. Song, F. Yu and Y.H. Li, "Mechanical property of synthesized methane hydrate under triaxial compression", J. Natural Gas Chem., vol. 19, pp.246-250, 2010.

[38] K. Miyazaki, A. Masui, Y. Sakamoto, K. Aoki, N. Tenma and T. Yamaguchi, "Triaxial compressive properties of artificial methanehydrate-bearing sediment", J. Geophys. Res., vol. 116, pp. B06102, 2011.

[39] J.L. Briaud and A.J. Chaouch, "Hydrate melting in hydrate soil around hot conductor", J. Geotech. Geoenviron. Eng., vol.123, no. 7, pp.645-653, 1997.

[40] C.Ji, G.Ahmadi and D. H.Smith, "Constant rate natural gas production from a well in a hydrate reservoir",Energy Convers. Manage., vol. 44, no.15, pp.2403-2423, 2003.

[41] D. Huang, S. Fan, "Measuring and modeling thermal conductivity of gas hydrate-bearing sand", J. Geophys. Res., vol.110, no.B1, pp.B01311, 2005.

[42] Grevemeyer and H. Villinger, "Gas hydrate stability and the assessment of heat flow through continental margins", Geophys. $J$. Int., vol. 145, no. 3, pp.647-660, 2001.

[43] G. R. Dickens, J. R. Oneil, D. K. Rea and R. M. Owen, "Dissociation of oceanic methane hydrate as a cause of the carbon-isotope excursion at the end of the paleocean", Paleoceanography, vol.10, no.6, pp. 965-971, 1995.

[44] W.Y. Xu and C. Ruppel, "Predicting the occurrence, distribution, and evolution of methane gas hydrate in porous marine sediments", J. Geophys. Res., vol.104, no.B3, pp. 5081-5095,1999.

[45] P. Kumar, D. Turner and E.D. Sloan, "Thermal diffusivity measurements of porous methane hydrate and hydrate-sediment mixtures", J. Geophys. Res.,vol.109, no.B1, pp.B01207, 2004.
[46] W.F. Waite, B.J.de Martin, S.H. Kirby, J. Pinkston and C. D. Ruppel, "Thermal conductivity measurements in porous mixtures of methane hydrate and quartz sand", Geophys. Res. Lett., vol.29, no.24, pp. 2229, 2002.

[47] D. C. Douglas, A. I. Martin, T. S. Yun, F. M. Francisca, J. C. Santamarina and C. Ruppel, "Thermal conductivity of hydrate-bearing sediments", J. Geophys. Res., vol.114, pp. B11103,2009.

[48] D. Z. Huang and S. S. Fan, "Thermal conductivity of methane hydrate formed from sodium dodecyl sulfate solution", J. Chem. Eng. Data, vol. 49, no. 5, pp.1479-1482, 2004.

[49] W. F. Waite, L. A. Stern, S. H. Kirby, W. J. Winters, D. H. Mason, "Simultaneous determination of thermal conductivity, thermal diffusivity and specific heat in sI methane hydrate", Geophys. J. Int., vol. 169, no. 2, pp.767-774, 2007.

[50] Ruppel and M. Kinoshita, "Fluid, methane, and energy flux in an active margin gas hydrate province, offshore Costa Rica", Earth Planet. Sci. Lett., vol. 179, no. 1, pp.153-165, 2000.

[51] M.B. Rydzy, J.M. Schicks, R. Naumann and J. Erzinger, "Dissociation enthalpies of synthesized multicomponent gas hydrates with respect to the guest composition and cage occupancy", J. Phys. Chem. B, vol.111, no.32, pp.9539-9545, 2007.

[52] S. Circone, S.H. Kirby, L.A. Stern, "Direct measurement of methane hydrate composition along the hydrate equilibrium boundary", J. Phys. Chem. B, vol.109, no.19, pp.9468-9475,2005.

[53] D.J. Turner, P. Kumar, E.D. Sloan, "A new technique for hydrate thermal diffusivity", Int. J. Thermophys., vol. 26, no.6, pp.1681$1691,2005$.

[54] J. Nimblett, C. Ruppel, "Permeability evolution during the formation of gas hydrates in marine sediments", J. Geophys. Res., vol 108, no. B9, pp.B001650, 2003.

[55] Y. Sakamoto, T. Komai, T. Kawamura, N. Tenma and T. Yamaguchi, "Field scale simulation for the effect of relative permeability on dissociation and gas production behavior during depressurization process of methane hydrate in marine sediments", In: Proceedings of the $7^{\text {th }}$ International Offshore and Polar Engineering Conference, Ocean Mining Symposium, 2007, pp. 102-107.

[56] H. Minagawa, Y. Nishikawa, L. Ikeda, Y. Sakamoto, T. Komai and T. Narita, "Measurement of methane hydrate sediment permeability using several chemical solutions as inhibitors", In: Proceedings of the $7^{\text {th }}$ International Offshore and Polar Engineering Conference, Ocean Mining Symposium, 2007, pp. 87-92.

[57] X. L. Liu and P. B. Flemings, "Dynamic multiphase flow model of hydrate formation in marine sediments", J. Geophys. Res., vol.112, no.B3, pp.B03101, 2007.

[58] H. Minagawa, Y. Nishikawa, I.Ikeda, K. Miyazaki, N. Takahara, Y. Sakamoto, T. Komai and T. Narita, "Characterization of sand sediment by pore size distribution and permeability using proton nuclear magnetic resonance measurement", J. Geophys. Res., vol.113, pp.B07210, 2008.

[59] R. L. Kleinberg, C. Flaum, D. D. Griffin, P. G. Brewer, G. E. Malby, E. T. Peltzer and J. P. Yesinowski, "Deep sea NMR: methane hydrate growth habit in porous media and its relationship to hydraulic permeability, deposit accumulation, and submarine slope stability", J. Geophys. Res., vol.108, no.B10, pp.2508, 2003.

[60] M.W. Lee and W.F. Waite, "Estimating pore-space gas hydrate saturations from well log acoustic data",. Geochem. Geophys. Geosyst., no.9, pp.Q07008, 2008.

[61] Y. Soel and T.J. Kneafsey, "Methane hydrate induced permeability modification for multiphase flow in unsaturated porous media", $J$ Geophys. Res., vol.116, pp.B08102, 2011..

[62] H. C. Amyx, D. M. Bass and R. L.Whiting, Petroleum reservoir engineering physical properties, McGraw-Hill Book Co.: New York, 1960.

[63] A. E. Cook, D. Goldberg and R. L. Kleinberg, "Fracture-controlled gas hydrate systems in the northern Gulf of Mexico", Mar. Petrol. Geol., vol.25, pp.932-941, 2008.

[64] Y. Jin, J. Hayashi and J. Nagao, K. Suzuki, H. Minagawa, T. Ebinuma and H. Narita, "New method of assessing absolute permeability of natural methane hydrate sediments by microfocus X-ray computed tomography", Jpn. J. Appl. Phys., vol. 46, no. 5A, pp.3159-3162, 2007.

[65] P.C. Robinson, "Connectivity of fracture systems: a percolation theory approach", J. Phys. A. Math. Gen., no.16, pp.605-14, 1983.

[66] H.P. Xie, Introduction to fractal and rock mechanics, Science Press, Beijing, 1996, (in Chinese) 
[67] H. Alkan, "Percolation model for dilatancy-induced permeability of the excavation damaged zone in rock salt", Int. J. Rock Mech. Min. Sci., vol.46, no.4, pp.716-24, 2009.

[68] Stauffer and A. Aharony, Introduction to percolation theory, Taylor \& Francis: London, 1992.

[69] M. Sahimi, Application of percolation theory, Taylor \& Francis, London, 1993.

[70] A. Bunde, and S. Havlin, Fractals and disordered system, Springer-Verlag, Berlin, 1996.

[71] W. Zheng, X.B. Lu, and Q. P. Liu, "Study on the connection of fractured pore media based on dual-percolation model", Chin. J. Rock Mech. Eng., vol.30, no.6, pp.1289-1296, 2011(in Chinese).

[72] Fatt, "The network model of porous media I. Capillary pressure characteristics", Trans. AIME, vol.207,pp.144-159, 1956a.

[73] Fatt, "The network model of porous media II. Dynamic properties of a single size tube network", Trans. AIME, vol. 207, pp.160$163,1956 \mathrm{~b}$.

[74] Fatt, "The network model of porous media III. Dynamic properties of networks with tube radius distribution", Trans AIME, vol.207, pp.164-181, 1956c.

[75] X.H. Zhang, Q.J. Liu, and X.B. Lu, "Comparisons of static, quasistatic and dynamic 3D porous media scale network models for two phase immiscible flow in porous media", In: Proceedings of the Fifth International Conference on Fluid Mechanics, 2007, pp.530533.

[76] X. B. Lu, W.Zheng, Q. J. Liu and X. H. Zhang, "Permeability of fractural porous media determined by double percolation model", Open Numer. Methods J., no. 3, pp.1-6, 2011.

[77] T.J. Kneafsay, Y. Soel, A. Gupta and L. Tomutsa, "Permeability of laboratory formed methane hydrate-bearing sand: measurements and observations using X-raycomputed tomography", SPE J., vol.16, no.1, pp.78-94, 2011.

[78] J.M. Carcione and D. Gei, "Gas-hydrate concentration estimated from P- and S-wave velocities at the Mallik 2L-38 research well, Mackenzie Delta", Can. J. Appl. Geophys., vol.56, pp.73-78, 2004.

[79] K. Weitemeyer, C. Conatable, K. Key and K. Key, "Marine EM techniques for gas-hydrate detection and hazard mitigation", Lead. Edge, vol.25, no.5, pp.629-632, 2006.

[80] F. Coren, V. Volpi and U. Tinivella, "Gas hydrate physical properties imaging by multi-attribute analysis--Blake Ridge BSR case history", Mar. Geol., vol.178, pp.197-210, 2001.

[81] S. Rajput, P.P. Rao, and N.K. Thakur, "Two dimensional elastic anisotropic/AVO modeling for the identification for the BSRs in marine sediments using multicomponent receivers", Geo. Mar. Lett., vol. 25, pp.241-247, 2005.

[82] B.L. Whiffen, H. Kiefte and M.J. Clouter, "Determination of acoustic velocities in xenon and methane hydrates by Brillouin spectroscopy", Geophys. Res. Lett., no. 9, pp.645-648, 1982.

[83] M. Bathe, S. Vagle, G.A. Saunders and E. F. Lambson, "Ultrasonic wave velocities in the structure II clathrate hydrate THF17H20", Mater. Sci. Lett., no.3, pp.904-906, 1984.

[84] H. Kiefte, M.J. Clouter and R.E. Gagnon, "Determination of acoustic velocities of clathrate hydrates by Brillouin spectroscopy", J. Phys. Chem., vol.89, pp.3103-3108, 1985.

[85] R.J. Wittebolle, and D.C. Sego, "A laboratory facility for testing sediments containing gas hydrates", In: Proceedings of the $4^{\text {th }}$ International Offshore Mechanics and Arctic Engineering Symposium, 1985, pp.52-58.

[86] C.F. Pearson, J. Murphy and R. Hermes, "Acoustic and resistivity measurements on rock samples containing tetrahydrofuran hydrates: Laboratory analogues to natural gas hydrate deposits", Geophys. Res., vol.91, pp.14132-14138, 1986.

[87] X. Su, C. B. Song and N. Q. Fang, "Variation in grain size of sediments above BSR and correlation with the occurrence of gas hydrate on hydrates ridge", East Pac. Earth Sci. Front., vol. 12, no. 1, pp.234-242, 2005

[88] E.C. Willoughby, K. Latychev, R.N. Edwards and G. Mihajlovic, "Resource evaluation of marine gas hydrate sediments using seafloor compliance methods", Ann. N. Y. Acad. Sci., vol.912, pp.146-158, 2000 .

[89] K. Andreassen, P.E. Hart, and M. Mackey, "Amplitude versus offset modeling of the bottom simulating reflection associated with submarine gas hydrate", Mar. Geol., vol.137, pp.25-40, 1997.

[90] S .M. Lu, and G..A. McMechan, "Elastic impedance inversion of multichannel seismic data from unconsolidated sediments contain- ing gas hydrate and free gas", Geophysics, vol.69, no.1, pp.164179,2004

[91] F. L. Tang, S. Z. Zhang, G. S. Jiang, X .Y. Liu, and B. Du, "Introduction to coring technology by drilling of gas hydrate", Geol. Sci. Technol. Inform., vol.21, no.2, pp. 97-99, 2002. (in Chinese)

[92] G.R. Dickens, C.K. Paull, and P. Wallace, "Direct measurement of in situ methane quantities in a large gas-hydrate reservoir", Nature, vol.385, no.6615, pp.426-428, 1997.

[93] M. Mathews, "Logging characteristics of methane hydrate", Log Analyst, vol.27, no.3, pp.26-63, 1986.

[94] J.A. Priest, A.I.Best and C.R.I.Clayton, "A laboratory investigation into the seismic velocities of methane gas hydrate-bearing sand", $J$. Geophys. Res., vol.110, pp.B04102, 2005.

[95] J.A.Priest, A.I.Best and C.R.I.Clayton, "Attenuation of seismic waves in methane gas hydrate-bearing sand", Geophys. J. Int., vol.164, pp.149-159, 2006.

[96] S. Y. Wang and X. B.Lu, "Recent advances in techniques of and analytical methods of deepwater geotechnical investigations", Ocean Eng., vol.259, no.2, pp.126-130, 2007, (in Chinese).

[97] J. Zhang, Y.G.. Ye, S.B.Xi and C .L. Liu, "Application of altrasonic detecting technology in the experimental study of gas hydrate", Geosicence, vol.19, no.1, pp.113-118, 2005, (in Chinese).

[98] D.Gei, and J.M. Carcione, "Acoustic properties of sediments saturated with gas hydrate, free gas and water", Geophys. Prospect., vol.51, no.2, pp.141-157, 2003.

[99] M.B.Helgerud, J.Dvorkin and A.Nur, "Rock physics characterization for gas hydrate reservoirs. Gas Hydrate Reservoirs: Elastic Properties", Ann. N. Y. Acad. Sci., vol. 912, pp.49-58, 2000.

[100] N.L.B.Bangs, D.S.Sawyer and X.Golovchenko, "Free gas at the base of the gas hydrate zone in the vicinity of the Chile triple junction", Geology, vol.21, pp.905-908, 1993.

[101] W.T.Wood, P.L.Stoffa and T.H.Shipley, "Quantitative detection of methane hydrate through high resolution seismic velocity analysis", J. Geophys. Res., vol.99, no. B5, pp.9681-9695,1994.

[102] G, Guerin, D. Goldberg and A. Meltser, "Characterization of in situ elastic properties of gas hydrate-bearing sediments on the Blake Ridge", J. Geophys. Res., vol.104, no.B8, pp. 17781-17795, 1999.

[103] M.B. Helgerud, J. Dvorkin, A. Nur, "Rock physics characterization for gas hydrate reservoirs-elastic properties", Ann. N. Y. Acad. Sci., vol. 912, pp.116-125, 2000.

[104] A. Sakai, "Can we estimate the amount of gas hydrate by seismic methods", Ann. N. Y. Acad. Sci., vol.912, pp.374-391, 2000.

[105] R.D. Hyndman and G.D. Spence, "A Seismic study of methane hydrate marine bottom simulating reflectors", J. Geophys. Res.,vol. 97, no.B5, pp.6683-6698, 1992.

[106] C.F.Pearson, P.M.Halleck, P.L.McGure, R. Hermes and M. Mathews, "Natural gas hydrate: A review of in situ properties", $J$. Phys. Chem. vol.87, pp. 4180-4185, 1983.

[107] M.W. Lee, D.R. Hutchinson, T.S. Collet and W .P. Dillon, "Seismic velocities for hydrate- bearing sediments using weighted equation", J. Geophys. Res., vol.101, no.B9, pp.20347-20358, 1996.

[108] M.W. Lee and T.S. Collett, "Elastic properties of gas hydratebearing sediments", Geophysics, vol.66, no.3, pp.763-771, 2001.

[109] M.W. Lee, "Biot-Gassmann theory for velocities of gas hydratebearing sediments", Geophysics, vol.67, no.6, pp.1711-1719, 2002.

[110] M. Jakobsen, J.A. Hudson, T.A. Minshull and S. C. Singh, "Elastic properties of hydrate-bearing sediments using effective medium theory" J. Geophys. Res., vol.105, no.B1, pp.561-577, 2000.

[111] Toyasa, and A. Nur, "Effects of diagenesis and clay on compressional velocities in rock", Geophys. Res. Lett., vol.9, pp.5-8, 1982.

[112] A.B. Wood, A textbook of sound, G. Bell and Sons, London, p.578, 1941

[113] D.C. Nobes, H. Villinger, F.F. Davis and L. K. Law, "Estimation of marine sediment bulk physical properties at depth from seafloor geophysical measurements", J. Geophys. Res., vol. 91, pp.1403314043,1986

[114] M.A. Biot, "Theory of propagation of elastic waves in a fluid saturated porous solid. I. Low frequency range; higher frequency range", J. Acoust. Soci. Am., vol. 28, pp.168-191,1956.

[115] M.A. Biot, "Generalized theory of acoustic propagation in porous dissipative media", J. Acoust. Soci. Am., vol.34. pp.1254-1264, 1962 .

[116] Gassmann, "Elasticity of porous media", Vierteljahrsschr, Naturforsh. Ges., vol.96, pp.1-23, 1951

[117] D.R. Murray, R.L. Kleinberg, B.K. Sinha, M. Fukuhara, O. Osawa, T. Endo and T. Namikawa, "Saturation, acoustic properties, growth 
habit, and state of stress of a gas hydrate reservoir from well logs", Petrophysics, vol.47, no.2, pp. 129-137, 2006.
[118] S. Y. Wang and, X. B. Lu, "Recent advances in techniques of and analytical methods of deepwater geotechnical investigations", Ocean Eng., vol.25, no.2, pp. 126-130, 2007, (in Chinese).

(C) Lu et al.; Licensee Bentham Open.

This is an open access article licensed under the terms of the Creative Commons Attribution Non-Commercial License (http://creativecommons.org/licenses/by-nc/3.0/) which permits unrestricted, non-commercial use, distribution and reproduction in any medium, provided the work is properly cited. 